\title{
Research on the Application and Design of Haze Prevention Appliances for Outdoor Staffs
}

\author{
Mengfan $\mathrm{Hu}$ \\ China Jiliang University, Higher Education Campus of Xiasha in Hangzhou, 310018 \\ 517677657@qq.com
}

Keywords: Haze Prevention Product Design; Outdoor staffs; Haze Prevention Appliances; Industrial design

\begin{abstract}
With the development of modern industry, the pollution problem has become an inescapable problem for urban inhabitants throughout the world. Fog and haze pollution as a serious matter has brought great disaster to human beings and natural environment. This paper explores the impact of smog and haze as well as the prevention status of outdoor staff, characteristics, measures and development trend so as to introduce haze prevention series appliances design for outdoor staffs in smoggy weather. With the rapid economic development, people pay more and more attention to the environment which ignites their attention and recognition to their health. Therefore, problems for outdoor staffs to prevent and protect their health in bad weather with haze and fog have received sky rocketing attention. Besides, protective equipment is gradually accepted by people with requirements. This paper starts from the harmonious perspective between human being and animals to think about their realistic meaning in order to analyze the structure of haze prevention appliances; besides, it attempts to have a good knowledge of its practicality so as to penetrate into the psychological benefits, physical design to know the possibility of function expanding; moreover, it also analyzes the pursuit for haze prevention appliance to explore the new haze prevention appliance design which can meet the requirements of outdoor staffs.
\end{abstract}

\section{Effect of haze}

Haze, known as the killer of winter, has a bad effect on the respiratory tract of human beings while being attached to morning dew or drops along with industrial exhaust, car exhaust, dust, bacteria and virus in the air, which will ignite upper respiratory infection (cold), acute tracheobronchitis, pneumonia, bronchial asthma, and chronic bronchitis etc. Besides, foggy air quality is poor, diabetic patients with poor resistance maybe affected in terms of lung and respiratory, leading to progress of case. In the following, let's have a basic knowledge of hazards of haze:

Effect on respiratory system. Haze which will cause bad effect on health mainly refers to aerosol particles with diameter less than 10 microns such as particles, sea salt, sulfate, nitrate, organic aerosols, fuels and vehicle emissions etc which will directly get into the body and alveolus pulmonis of human. Besides, submicron particle will be attached to upper and lower respiratory as well as alveolus pulmonis which will induce allergic rhinitis and bronchitis. For chronic respiratory disease patients with bronchial asthma, chronic bronchitis, obstructive pulmonary emphysema and chronic obstructive pulmonary disease, they may be greatly affected by the bad weather with fog and haze. Even worse, if they are exposed to the environment for a long time, they may have lung cancer. 
Effect on cardiovascular system. Smoggy days will also lead to cardiovascular disease, hider normal blood circulation and ignite cardiovascular disease, high blood pressure, coronary heart disease, stroke as well as stenocardia, heart attack, heart failure etc. In addition, it may lead to pulmonary heart disease. Moreover, the atmospheric pressure is relatively low in foggy days, people shall feel irritable and consequently the blood pressure shall be increased. Still, the temperature in foggy days is low, and some patients with hypertension, coronary heart disease suddenly go cold from warm may have stroke and myocardial infarction because of contraction of vascular.

Smoggy days can also lead to weakening of UV in ground layer, strengthen the activity of infectious germs in the air and increase infectious diseases.

Detrimental to children's growth. Because of sunshine reduction, children who are lack of ultraviolet radiation or vitamin D are in deficiency, while the absorption of calcium also greatly reduceswhich may cause rickets as well as decreased growth.

Effect on mental health. Experts pointed out that continuous foggy days have impact on people's psychology and body. Judging from psychology, foggy days will give dull and depressing feelings which may irritate or exacerbate depression. In addition, fog light is weak which will lead to low pressure and some people will feel lazy and depressed.

\section{It will also affect reproductive ability.}

Passive effect on climate, environment, health, and economy etc. Phenomena such as acid rain, photochemical smog will lead to visibility reduction and hold up the air, water and land surface traffic. What's more, it will also aggravate chronic disease, respiratory system and cardiac system diseases, change the lung function and structure, affect reproductive ability and change the immune system of human body.

Effect on the traffic safety. In foggy days, the visibility is low with bad quality of air, which may lead to traffic jams and congestion and cause traffic accident.

\section{Foggy days easily cause cancer.}

\section{Present situation of haze prevention of outdoor staffs}

After carrying out field surveys and interviews of outdoor staffs, they only wear cottony or disposable mask with weak barrel properties. Some outdoor workers wear gloves or hats, which have poor function in terms of preventing bacteria or dust.

\section{Feature analysis of outdoor staffs}

Analysis on job characteristics: outdoor workers need to work outside for a long time with almost 8 hours of working time per day from early morning until late at night, experiencing all major periods. Regardless of season, rain or polluted environment, they are still exposed to the sun as the air to complete the daily work. Main outdoor jobs are traffic police, road cleaning, site builders, and so on. By the way, their working places are roads, construction sites etc.

Analysis on psychological needs: with the pollution becoming more aggravated day by day, outdoor staffs pay special attention to their health. Because of work specificity, they have to be exposed to outdoor air and atmospheric pollution for a long time. Therefore, they are easily affected by environmental problems. So they shall ask higher requirements on the haze prevention appliances than common people. They expect that the haze prevention appliances can help to decrease the band effect of air pollution on their body. 
Analysis on dependent demand: The wearing of protection equipment is effective to deal with the bad weather and outdoor staffs particularly depend on the wearing and prevention of protective equipment.

\section{Analysis on the haze prevention measures of outdoor staffs}

The most effective way to prevent haze is to wear haze prevention masks. Surgical masks can prevent $95 \%$ of the particle with 0.3 micron diameter, which can effectively avoid the infection on respiratory tract. However, you have to choose qualified masks and appropriate size to be close to your skin and prevent the entering of contamination particle. Masks cannot be washed yet should be fold down when it is dry so as to avoid the microorganism breeding. The point which is worth being paid attention to is that the old and patients with cardiovascular disease cannot wear masks because they completed covered the face which may cause it is hard to breeze and people will feel dizzy due to lack of oxygen.

Now, there is a dazzling array of dust prevention masks and most of these are disposable products. The use period for disposable products cannot over 4 hours. However, for cities with serious problems in term of air pollution, outdoor staffs work more than 4 hours outside. That is to say, every outdoor staff has to wear more than 2 masks if they are exposed to the fog and haze.

Existing non-disposable masks are not easy to take and clean without a good ability in terms of dust prevention. So these kinds of masks cannot meet the wearing requirement of outdoor staffs.

The nose should be paid vital attention to while wearing masks. As we all know, inhalable particles as well as dust pollution floating in the air cause harm on human body through respiratory and nasal breathing is of vital importance. Therefore, in daily prevention, the nose protection should not be ignored and people work outside cannot only cover their mouth and nose when they are exposed.

Existing protective masks cannot play a good role in protecting the nose which is also not comfortable while wearing.

Outdoor staffs should wear eyeshades when it is heavily polluted. Survey results show that 99\% bacteria and pollutants in the air can enter into the body through the cornea so as to infect the human health. Therefore, if it is heavily polluted, outdoor staffs have to pay attention to eyes if they are exposed to the air environment for a long time.

Focus on body prevention. Most people think that it is enough to pay attention to eyes, nose and mouth to prevent the haze. But in fact, most bacteria and other contaminants have already be attached to you when you are outside. When you get home, you are bringing these organisms which may lead to secondary infections while taking off the prevention masks. Therefore, it is necessary to focus on physical protection.

\section{The future development trend of haze prevention appliances}

Haze prevention appliances, as the name suggests, aim to prevent the body while facing the increasingly serious environment. Sky rocketing people (especially outdoor staffs) pay attention to the convenience, aesthetic and material change while taking the efficiency into consideration.

At present, haze prevention products are simple and cute affected by Nordic design, postmodern, Japanese and Korean culture. The design in the future has the following features: 
Practicability: The measurement criteria for product are mainly reflected in the practicability, and haze prevention appliances are no exception. While purchasing products, the first point should be paid attention to is the practicability. In fact, it is not difficult to find out that products which have a good sales volume are mainly designed based on practicability regardless of the material and function. By the way, we have to explore other features to enrich the appearance, shape, color, and so on.

Legibility: the following shall be comprehensively taken into consideration: production, use process and material selection. Finally, the harmony of product and human beings shall be taken into consideration. There are diversified materials and designers are able to have inspiration in the course of designing so as to clear the point.

Integrity: Protection products are not only confined to the local protection, which should also include the mouth and nose or eye protection. Besides, it contains the three stages of wearing, using and taking off.

Personalization: Everyone has different experiences toward life, it is not impossible to figure out perfect masks, and designers have to consider from various perspectives and judge standing in other people's shoes.

\section{The design of haze prevention appliances for outdoor staffs}

\section{Design Renderings}

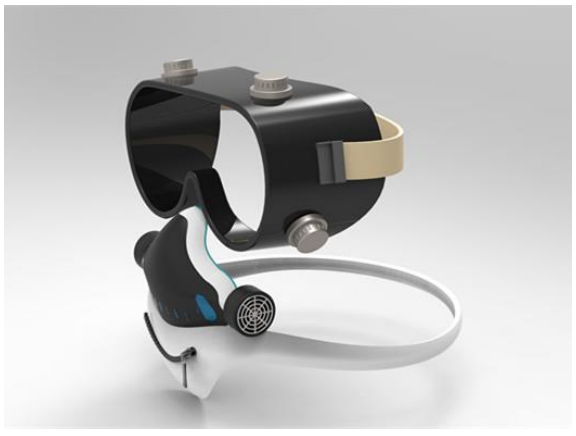

protective mask

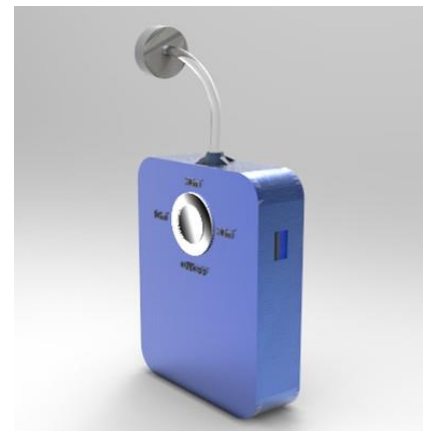

air cleaner

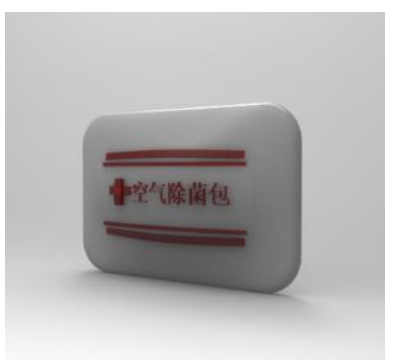

Air sterilization bags

Design philosophy. The design includes eye protecting mask, nose cup, air purifier, air sterilization package. Eye protecting mask is separated from the nose cup which can meet the requirement of comfortability for fixed groups.

Outdoor workers mainly work depending on hand so the air sterilization package should be tiny which should be convenient to carry. They can put it into the pocket or purse so as to not prevent their daily work. The content in air sterilization package is solid chlorine dioxide, which can eliminate the influenza germs in the air and PM2.5 etc. Working principle: it has the ability to suppress the virus, putrefying bacteria and mould etc. which can be put into the bag to protect the air within 1 meter range.

The part in the mouth position of protective mask takes cotton as material, which is easy to fold and carry. The zipper can help people to eat, drink etc. In terms of oronasal mask, the design is made of elastic. Besides, the curve is designed based on the structure of people's nose and the distance between the plastic mask and face is appropriate and the mask fits the skin completely with distances for people to breeze so as to keep smooth breathing. 
The interface part of portable air purifier is connected to the air-vent of the nose. When it is heavily polluted or outdoor staffs have special needs, the air cleaner can be open so as to have fresh air. The tube which is attached to off-gas outlet is retractable and portable.

The blinker is designed thick which can block the light and avoid the harm on eye while working in the environment with bright sunlight. Four knobs can be adjusted or taken off for the requirement of outdoor staffs.

The program satisfactorily solves the haze prevention appliances for outdoor workers to protect themselves which are convenient to wear and carry.

\section{Conclusion}

At present, the industrial design is developed quickly and people's consciousness toward environment protection as well as health is gradually enhanced. As a result, the haze prevention will receive sky rocketing attention. The haze prevention appliances design is a comprehensive circle, involving a glittering array of knowledge. More careful exploration and research on details shall be further carried out so as to find out the best combination of points for different people based on the relation between design and technology.

\section{References}

[1] Cui Hongyan. A Brief Discussion on Haze. Resources Economization \& Environment. 7th Term of 2013;

[2] Karis Baldwin, Kim Clark. Design Rules: The Force of Modularization. Beijing: Citic Press Corp, 2006:78;

[3] Zou Xiaosong, Wang Nianwen. The Visual Communication of Product Design in Terms of Form, Color and Quality [J]. Art Panorama, ,2007, (01 ):106-107;

[4] Ma Mingyuan, Chen Meiyu, Wang Dan, She Dongdong. The Development Situation of Future of Breathing Mask. Progress in Textile Science \& Technology, 6th Term of 2014

[5] Wu Xiaoqi. Comparative Observation on the Service Performance of 3M Dust Masks used for Mine and Domestic Common Masks [J] Chinese Journal of Clinical Rational Drug Use; 19th Term 2012

[6] Li Wanbin, Zhong Xinhua, Wu Zhou, Li Huimei. Fitness Test for Masks. Applied Journal of General Practice, 9th term of 2010

[7] Lin Guilan. Break the Rules of Design. Sanlian bookstore Press. March 2007

[8] Laura Slack. What is Design. China Youth Press. January 2008

[9] Chen Shixin.The Characteristics and Application of Surgical Masks[J] Nursing Practice and Research; 19th Term 2010

[10] Wang Xiding.Dust and Harm.[J] Glass; 2th Term 1997 